\title{
TEXTURE EVOLUTION OF "AMARETTI" COOKIES DURING STORAGE
}

\author{
Antonio Piga*, Pasquale Catzeddu1, Stefano Farris, Tonina Roggio2, Annamaria Sanguinetti3, \\ Efisio Scano4 \\ Dipartimento di Scienze Ambientali Agrarie e Biotecnologie Agro-Alimentari, Viale Italia 39/A, \\ 07100 Sassari, Italy \\ 1 Porto Conte Ricerche Soc. Cons a r. 1., Località Tramariglio - 07041 Alghero, Italy \\ 2 POLARIS, Parco scientifico e tecnologico della Sardegna, Località Tramariglio - 07041 Alghero, \\ Italy \\ 3 External collaborator, Via Paolo Conida 3, 07014 Ozieri (SS) \\ 4 Consultant, Viale Rinascita 15, 09025 Sanluri (Ca), Italy
}

*To whom correspondence should be addressed. Tel: +39 079-229273; fax: +39 079-229273; email:pigaa@uniss.it.

\begin{abstract}
The results of a study on texture evolution during 35 days of storage of "amaretti", a typical Italian cookie, packaged in two different ways are reported. Amaretti cookies were wrapped in polyvinylchloride (PVC) film or aluminium foil (ALL), to simulate two different permeability conditions and stored at controlled temperature and humidity. Evolution of texture (such as hardness) and aw were tested instrumentally by a texture analyser and a hygrometer, respectively. Texture was assessed by a cut and puncturing test. Indices for hardening were area under the curve $(\mathrm{N} x \mathrm{~mm})$ and gradient $(\mathrm{N} / \mathrm{mm})$ for the puncturing test and maximum force $(\mathrm{N})$ for the cut test. Both textural tests showed significantly higher hardening of PVC cookies, compared to the ALL cookies. The latter retained good sensorial properties at the end of the storage period, although their internal structure changed from soft and moist to mealy, while the PVC cookies were no longer edible only 10 days after baking. Aw values decreased and increased in PVC and ALL lots, respectively. The results suggest that hardening may be explained by water loss in PVC and moisture redistribution in ALL.
\end{abstract}

Running title: hardening of cookies

List of abbreviations: ALL, PVC, ERH

Key words: Cookies, hardening, structure, sugar crystallisation, texture analysis TEXTURE EVOLUTION OF "AMARETTI" COOKIES DURING STORAGE

\section{Introduction}

Cookies are characterised by moisture and water activity (aw) higher than $7 \%$ and 0.5 , respectively [1]. The short is usually a mixture of several ingredients, made according to a fairly complex recipe and in a short time [2]. Cookies have the capacity of bending after baking, when they are fresh, unlike biscuits that break when bent. On the other hand, biscuits usually have below 5\% moisture content, with water activity of around 0.2. This may lead to absorption of water from the atmosphere following prolonged exposure to ambient conditions, making the biscuit soft and soggy [2], thus packaging with a moisture impermeable film is a common practice. In contrast, hardening is the main cause of quality deterioration of cookies, which change from soft and pliable to firm and crumbly within a few days or even hours after baking. While biscuit shelf-life has been extensively studied, little research has been done on cookies. Recently, a very extensive work was published on the web on the textural evolution of cookies during storage [1]. In this paper the extent of sugar crystallisation in lab-made cookies was determined by replacing part of the sucrose with high fructose corn syrup (HFCS) or trehalose. The authors concluded that, although a strong correlation between cookie hardening and sucrose crystallisation does exist, the two sugar substitutes reduced the firming process while not inhibiting or only slightly retarding the crystallisation rate. Thus, 
contrary to what has been previously reported [3], sucrose crystallisation is probably not the only cause of texture deterioration of cookies. Hardening, in any case, is probably a direct consequence of water redistribution among components and loss of plasticizer volume, as reported in another study [4]. The authors concluded that both HCFS and trehalose may act as plasticizers. More simply, it cannot be excluded that hardening is a consequence of water loss from the cookie surface.

"Amaretti" are typical Italian cookies, found in several regions of Italy. In general, amaretti cookies are made of sweet and bitter almonds, sucrose and egg white. Amaretti means bitter cookies, because of the taste given by the bitter almonds. Freshly baked amaretti are soft and delicious but undergo severe hardening after seven to ten days, as observed in our lab. This problem strictly limits the shelf-life, so amaretti can be marketed only locally. To our knowledge, there are no studies dealing with the evolution of texture of amaretti cookies. .

With this in mind, we decided to conduct a preliminary investigation on the evolution of structure of freshly baked amaretti cookies, some packaged at water barrier conditions and some not, and stored at controlled temperature and equilibrium relative humidity (ERH).

2 Materials and methods

2.1 Amaretti cookies preparation

Amaretti cookies (amaretti from now on) were kindly provided by a local baker. Ingredients were in the following amounts:

- $\quad$ Sweet almonds $(0.75 \mathrm{~kg})$

- $\quad$ Bitter almonds $(0.25 \mathrm{~kg})$

- $\quad$ Sucrose $(1.0 \mathrm{~kg})$

- $\quad$ Egg white $(0.3 \mathrm{~kg})$

Ingredients were mixed together for 8 minutes at low speed with a vertical axis mixer, the short rested for 20 minutes, then formed into small discs of about $8 \mathrm{~cm}$ in diameter. Discs were cooked for 20 minutes at $180^{\circ} \mathrm{C}$, cooled at ambient temperature and packaged.

\subsection{Packaging and storage}

The amaretti were divided in two lots, one packaged (in groups of three) manually in PVC film, the other in ALL foil, the latter being impermeable to water, while the former is not. These two different materials were chosen in order to assess whether hardening can be ascribed to water loss from the amaretti (PVC) or from a redistribution of the moisture inside the amaretti (ALL) or by a combined mechanism of loss plus redistribution (PVC). Extreme care was taken to leave a minimum head space. The packaged amaretti were stored at $20^{\circ} \mathrm{C}$ and $40 \% \mathrm{ERH}$.

\subsection{Textural determinations}

Hardness and its evolution were determined in freshly baked amaretti and at 3, 7, 10, 14, 17, 21, 24, 28, 31, and 35 days, by using a texture analyser (mod. TA-XT2, Stable Microsystems, Surrey, UK) with a $50 \mathrm{~kg}$ load cell. The Texture Expert program version 1.21 was used for data analysis. Textural determinations were made in six amaretti per each lot by using a blade set with knife edge for a cut test $(\bmod . \mathrm{HDP} / \mathrm{BS})$, and a $4 \mathrm{~mm}$ diameter cylinder probe $(\bmod . \mathrm{P} / 4)$, for a puncturing test. In both cases the amaretti were cut or punctured right through, in order to check whether any different structural characteristics were present inside or on the surface. To do this the contact plates were substituted with: a) a slotted blade insert, which acted as a guide for the blade while supporting the product; b) a confectionery holder, which allows complete penetration and withdrawal of the sample, equipped with a $6 \mathrm{~mm}$ diameter top and bottom hole for puncturing (we therefore performed a punch test and not a punch and die test). Parameters of each test are reported in Table 1. Samples for puncturing were placed centrally on the contact plate and secured on the heavy duty platform before the test commenced. Samples for cutting were placed centrally under the knife edge. Both the load cell and probes were calibrated before each test. Amaretti were 
selected to be $70+5 \mathrm{~mm}$ in diameter and $15+1 \mathrm{~mm}$ thick, in order to have a standardised sample. Hardness measurement of samples by puncturing involved (Fig. 2) plotting force (in N) versus distance (in $\mathrm{mm}$ ) and two parameters were calculated as a measure of hardness: a) area under the curve (as $\mathrm{N}$. mm) up to $15 \mathrm{~mm}$ of puncturing [5]; b) gradient (as $\mathrm{N} / \mathrm{mm}$ ) calculated on the first yield point of puncturing. The maximum force (as $\mathrm{N}$ ) was used as an index for the cut test (Fig. 1).

\subsection{Water activity and dry matter determinations}

Water activity was determined in six amaretti previously used for texture analysis, by an electronic hygrometer (model Aw-Win, Rotronic, equipped with a Karl-Fast probe), calibrated in the range 0.1-0.95 with solutions of LiCl of known activity [6]. All parts of the amaretti were tested. Water content was determined in a vacuum oven for $12 \mathrm{~h}$ at $70^{\circ} \mathrm{C}$ [7].

\subsection{Sensorial evaluation}

A sample of the amaretti was used for an informal sensorial evaluation by five untrained panellists, who were asked to judge acceptability of the cookies.

2.6 Statistical analysis

Data were subjected to one-way analysis of variance (ANOVA) using MSTAT-C software. In particular, data were grouped either by lots (ALL and PVC), to assess differences for each storage time, or by storage time, to check for differences within each lot during storage. Means, when appropriate, were separated by Duncan's multiple range test at $\mathrm{P}<0.01$.

\section{Results and discussion}

\subsection{Texture evolution}

Before discussing the evolution of the structure we need to consider the original structure of the amaretti resulting from the textural test. In the cut test (Fig. 1) the probe cut the cookie right through and the resulting graph has a cuspidate profile, resulting from the progressive increasing force during cutting. The maximum peak was registered at $15 \mathrm{~mm}$ from the start of cutting, that is the thickness of the cookie; after that there was a drastic force loss and the residual force registered was due to the friction of the blade passing through the cookie (the depth of cutting was fixed at $30 \mathrm{~mm}$ ). This means that the cookie does not fracture into two major pieces, as happens in biscuits. On the contrary, it breaks into a number of pieces, as can be seen in Fig.1, due to the porous structure. The curve seems like a type A one, as reported by Bourne [8]. Thus, we considered the maximum peak force as the index for hardening. However, we will also discuss eventual changes in the profile of the graph. We now come to the puncturing (Fig. 2). A higher force was registered at the beginning of penetration, which ended abruptly when the probe penetrated into the cookie, a few millimetres after application of the force. This means that the upper surface was harder than the remaining part. The curve seems like a type $\mathrm{C}$ one, as reported by Bourne [8].

The evolution of structure, as can be seen by the cut test, shows substantial differences between the PVC and ALL lots. The maximum force, in fact, had a very different evolution during storage, as it increased from $59 \mathrm{~N}$ in freshly baked cookies to $383 \mathrm{~N}$ in 28 day-old PVC ones (Tab. 2, see also Fig. 3 ), while it did not increase at all (on the contrary the values decreased until the 28th day in storage) in ALL cookies (Tab. 2 and Fig. 4), with the exception of the last inspection time. The maximum force values were always significantly different between the two amaretti lots (Table 2). It should be pointed out that, starting from the 14th day, the curve relative to ALL cookies shows a double hump (Fig. 4), corresponding more or less to the top and bottom part of the amaretti, probably as the samples became harder on the surface. This may be due to water migration from the outer to the inner part. We stopped testing on the PVC lot at 28 days because the hardness exceeded the instrument threshold. Moreover, the excessive hardness made the PVC cookies fracture into two pieces when cut. Thus, we can say that:

- PVC cookies hardened very quickly, probably due to a loss of water from the surface. 
- ALL cookies hardened to a lesser extent than PVC ones, and a probable water migration from the surface to the interior occurred.

Results from the puncturing test also showed substantial differences between the two lots. PVC cookies underwent progressive hardening, as can be seen from the data (Tab. 2) and the curve evolution (Fig. 5). In fact, both area and gradient values increased dramatically during storage. Curves show that hardening affected the whole amaretti thickness, and in any case they fractured into two pieces at 28 and 35 days. A different picture was seen for ALL cookies (Fig. 6). In fact, area and gradient values were significantly lower than those of PVC cookies (Table 2). In the puncturing test (Fig. 6), the maximum force was registered at the top of the cookie until the 14th day, after which the curve shows a similar trend to that observed in the cut test, that is, the appearance of a double hump, corresponding to the top and bottom part of the cookie. Finally, it can be noted that the ALL cookies' curve at seven days shows a flat profile, indicating that the applied force was the same throughout the whole thickness of the amaretti (Fig. 6). The same pattern can be observed in the ALL cookies' cut test (Fig. 4).

This may be due to a progressive migration of water from the surface to the inside of the amaretti, leading to a structure change in the inner part of the samples. The area and gradient values increased significantly also in ALL cookies during storage (although to a lesser extent than in PVC cookies). The panellists, who found that the inside of the ALL cookies changed from soft and moist to mealy, appreciated 35 day-old ALL cookies, while PVC cookies were no longer edible after 10 days.

\subsection{Water activity and dry matter changes}

Freshly baked cookies have an aw of 0.54 . PVC cookies underwent a slow and progressive decrease in aw, while ALL cookies showed opposite behaviour (Fig. 7). The first trend is probably due to the water loss from the cookie to the surrounding environment (PVC is not an impermeable material), while the second may be ascribed to sucrose crystallisation. In fact, water redistribution inside the cookies could promote sucrose crystallisation, which, in turn, releases water, thus increasing the aw values [9]. What could have happened in ALL cookies during storage (we prefer to stay on a hypothetical level before making more accurate investigations)? Water could have migrated within the cookie, with consequent probable sucrose crystallisation, which changed the internal structure of the cookie from soft to mealy. The starting aw and moisture values of amaretti seem reasonably to confirm the hypothesis of crystallisation. In fact, we are surely above monolayer status, while the aw value allows crystallisation to occur in less than 3 days [1]. The evolution of aw was confirmed by the change in dry matter content (Fig. 7). Both the aw and dry matter values differed significantly between PVC and ALL cookies.

\section{Conclusions}

Hardening is a major problem in the shelf-life of cookies. Our study showed that packaging in aluminium has completely solved the problem of hardening. Cookies packaged in aluminium probably underwent water redistribution, and this may have led to sucrose crystallisation, with consequent change from a soft and moist texture to a mealy one. The ALL cookies were sensorially appreciated after 35 days storage. However, aluminium should undoubtedly be substituted with a barrier transparent film (consumers prefer to see the product). Further studies need to be carried out to: a) confirm our hypothesis with more extensive investigations (NMR imaging, for example); b) block or retard the water redistribution by changing the recipe (as little as possible since the amaretti cookie is a typical product), for example by introducing an emulsifier.

\section{References}

[1] Labuza, T.P., Panda, F., Roos, Y., Sury, R., Roe, K., Sherwin, C., Puhek, T., Patist, A., Zoerb, A., In:

http://faculty.che.umn.edu/fscn/Ted_Labuza/PDF_files/Presentations/XRDCookiesFull.pdf. 
[2] Manley, D., in: D. Manley (Ed.), Biscuit, cookie and cracker manufacturing manuals, Manual 1, Woodhead Publishing Limited, Cambridge 1998, pp 5.

[3] Roos, Y., Karel, M., J. Food. Sci. 1991, 56, 38-43.

[4] Kulp et al., Starch, 1993, 43, 53-57.

[5] Papantoniou, E., Hammond E.W., Tsiami, A.A., Scriven, F., Gordon, M.H., Schofield, J.D., J. Agric. Food. Chem. 2003, 51, 1057-1063.

[6] Labuza, T.P., Acott, K., Tatini, S.R., Lee, R.Y., Flink, J., McCall, W., J. Food Sci. 1976, 41, 910

[7] AOAC, in: Helrich, K. (Ed.), Official Methods of Analysis, Analytical Chemists, Inc, Arlington, VI 1990, pp 795.

[8] Bourne, M.C., in: Sherman, P. (Ed.), Food Texture and Rheology, Academic Press, New York/London 1979, pp. 95-142.

[9] Labuza, T.P, Hyman, C.R., Trends Food Sci. Techn. 1998, 9, 47-55. 